\title{
Carrier-induced ferromagnetism in $p-\mathrm{Zn}_{1-x} \mathrm{Mn}_{x} \mathrm{Te}$
}

\author{
D. Ferrand, J. Cibert, ${ }^{*}$ A. Wasiela, C. Bourgognon, S. Tatarenko, and G. Fishman ${ }^{\dagger}$ \\ Laboratoire de Spectrométrie Physique, CNRS_Université Joseph-Fourier Grenoble Boîte Postal 87, \\ F-38402 St Martin d'Hères Cedex, France \\ T. Andrearczyk, J. Jaroszyński, S. Koleśnik, and T. Dietl \\ Institute of Physics, Polish Academy of Sciences, al. Lotników 32/46, PL-02-668 Warszawa, Poland \\ B. Barbara and D. Dufeu \\ Laboratoire de Magnétisme Louis Néel, CNRS, Boîte Postal 166X, F-38042 Grenoble-Cédex, France
}

(Received 1 August 2000; published 1 February 2001)

\begin{abstract}
We present a systematic study of the ferromagnetic transition induced by the holes in nitrogen doped $\mathrm{Zn}_{1-x} \mathrm{Mn}_{x}$ Te epitaxial layers, with particular emphasis on the values of the Curie-Weiss temperature as a function of the carrier and spin concentrations. The data are obtained from thorough analyses of the results of magnetization, magnetoresistance, and spin-dependent Hall effect measurements. The experimental findings compare favorably, without adjustable parameters, with the prediction of the Rudermann-Kittel-Kasuya-Yosida (RKKY) model or its continuous-medium limit, that is, the Zener model, provided that the presence of the competing antiferromagnetic spin-spin superexchange interaction is taken into account, and the complex structure of the valence band is properly incorporated into the calculation of the spin susceptibility of the hole liquid. In general terms, the findings demonstrate how the interplay between the ferromagnetic RKKY interaction, carrier localization, and intrinsic antiferromagnetic superexchange affects the ordering temperature and the saturation value of magnetization in magnetically and electrostatically disordered systems.
\end{abstract}

DOI: 10.1103/PhysRevB.63.085201

PACS number(s): 72.80.Ey, 75.50.Pp, 75.30.Hx, 75.50.Dd

\section{INTRODUCTION}

The possibility of controlling ferromagnetic interactions between the localized spins by the carriers, ${ }^{1-5}$ as well as the demonstration of efficient spin injection into a normal semiconductor, ${ }^{6-8}$ have recently renewed the interest in diluted magnetic semiconductors (DMS). ${ }^{9}$ If made functional at reasonably high temperature, ferromagnetic semiconductors would allow one to incorporate spin electronics into usual electronics, and even path the way to integrated quantum computers. ${ }^{10} \mathrm{Up}$ to now, the carrier-induced ferromagnetism has been observed in lead-salt materials, $\mathrm{Pb}_{1-x-y} \mathrm{Sn}_{y} \mathrm{Mn}_{x} \mathrm{Te},{ }^{1}$ and in molecular beam epitaxy (MBE)grown semiconductors with the zinc-blende structure: $\mathrm{In}_{1-x} \mathrm{Mn}_{x}$ As (Ref. 11) and $\mathrm{Ga}_{1-x} \mathrm{Mn}_{x} \mathrm{As}$, (Refs. 2 and 12) as well as in $p$-doped $\mathrm{Cd}_{1-x} \mathrm{Mn}_{x}$ Te quantum wells ${ }^{4}$ and $\mathrm{Zn}_{1-x} \mathrm{Mn}_{x}$ Te epilayers, ${ }^{5}$ in which the observation of the onsets of magnetic ordering in the temperature range between 1 and $3 \mathrm{~K}$ corroborated theoretical predictions. ${ }^{3}$ Thus, while promisingly high Curie temperatures, $T_{C}$ up to $110 \mathrm{~K},{ }^{2,12}$ are observed in the GaAs-based compounds, they are dramatically lower in the II-VI DMS structures studied so far. However, the II-VI compounds appear as model materials, in which localized spins and the holes can be introduced and controlled independently, and modulation-doped heterostructures are feasible, so that dimensionality effects can be examined.

In this paper, we present the phase diagram of nitrogendoped $p-\mathrm{Zn}_{1-x} \mathrm{Mn}_{x} \mathrm{Te}$, i.e., the dependence of the CurieWeiss $(\mathrm{CW})$ temperature $T_{\mathrm{CW}}$ on the Mn content $x$ and the hole concentration $p$. The values of $T_{\mathrm{CW}}$ and $x$ were obtained from magnetization measurements, while the hole densities were deduced from the Hall resistivity, measured under such conditions that the spin-dependent component is negligible, i.e., either at room temperature, or at low-temperature in a high magnetic field. We show that the observed values of $T_{\mathrm{CW}}$ are well described by a mean-field model, in which the hole-mediated exchange interactions are treated either in terms of Rudermann-Kittel-Kasuya-Yosida (RKKY) coupling mechanism, ${ }^{3}$ or by its continuous-medium limit, i.e., the Zener model, ${ }^{3,13,14}$ when the hole density is not too high. Accordingly, three parameters govern the carrier-induced ferromagnetism: the spin-carrier exchange integral $\beta$, the effective content of magnetic ions $x_{\text {eff }}$, and the spin susceptibility of the carrier liquid $\chi_{h}$. These three parameters are well known or can be readily evaluated for $p-\mathrm{Zn}_{1-x} \mathrm{Mn}_{x}$ Te. Our findings emphasize the importance of taking carefully into account the competition between ferromagnetic and antiferromagnetic interactions as well as the complex structure of the valence band in the calculation of $\chi_{h} \cdot{ }^{14}$ Finally, we discuss the case of lower doping, for which the onset of hole localization is clearly observed. We show that localization does not perturb significantly the magnitude of Curie-Weiss temperature, but reduces the saturation value of magnetization and gives raise to slow spin dynamics.

The heavily doped DMS studied here lie in-between the case of diluted magnetic metals, ${ }^{15}$ where the standard RKKY theory is applicable, and lightly doped DMS, ${ }^{9}$ for which carriers thermally excited to the band ${ }^{16}$ and interactions between bound magnetic polarons ${ }^{17}$ have been suggested as agents mediating the ferromagnetic coupling. Our results make it possible to identify differences and similarities between ferromagnetic III-V and II-VI material systems. Furthermore, the findings demonstrate how spin-orbit coupling, the com- 
peting antiferromagnetic interactions, and the presence of electrostatic disorder affect the carrier-induced ferromagnetism. The Stoner ferromagnetic instability in disordered conductors at low temperatures have recently been discussed theoretically. ${ }^{18,19}$ We hope that our results will stimulate a similar analysis for the system of localized magnetic ions coupled ferromagnetically by carriers at the boundary of the Anderson-Mott localization.

\section{SAMPLES AND EXPERIMENTAL SET-UP}

$\mathrm{Zn}_{1-x} \mathrm{Mn}_{x}$ Te:N layers were grown ${ }^{5}$ by MBE on a 800 $\mu$ m-thick (001) $\mathrm{Cd}_{0.96} \mathrm{Zn}_{0.04} \mathrm{Te}$ substrate, on which a $300 \mathrm{~nm}$ CdTe and $200 \mathrm{~nm}$-thick ZnTe buffer layers were deposited, the latter much thicker than the critical thickness of the $\mathrm{ZnTe} / \mathrm{CdTe}$ system. The active layer, $\mathrm{Zn}_{1-x} \mathrm{Mn}_{x} \mathrm{Te}: \mathrm{N}$, typically $500 \mathrm{~nm}$ thick, was grown at $300^{\circ} \mathrm{C}$. The thickness of the active layer was estimated from the growth rate. Our accuracy is usually around $1 \%$ to $2 \%$. The $\mathrm{Zn}_{1-x} \mathrm{Mn}_{x}$ Te layers were deposited either by using the stoichiometric $\mathrm{Zn} / \mathrm{Te}$ flux from a $\mathrm{ZnTe}$ load and simply adding a Mn flux during the growth of the active layer (resulting in rather rough surfaces), or by using a $\mathrm{Zn}$ rich flux (adding an excess of $\mathrm{Zn}$ from an additional $\mathrm{Zn}$ cell, approximately $50 \%$ of the $\mathrm{Zn}$ flux from the ZnTe cell), which gives a smooth surface. A homedesigned electron cyclotron resonance (ECR) plasma cell served as a source of atomic nitrogen. ${ }^{20}$

Resistivity and Hall effect measurements were performed between room temperature and $1.5 \mathrm{~K}$ in a magnetic field $H_{o}$ up to $110 \mathrm{kOe}$, applied in the direction perpendicular to the film surface. In this geometry, there is no magnetization corrections to the external magnetic field acting on the carriers, $B=H_{o}$. At the same time, the field acting on the localized spins is diminished by the demagnetization correction, $H$ $=H_{o}-4 \pi M$. For $x=0.015$, the field produced by the saturated $\mathrm{Mn}$ spins is $B_{s}=4 \pi M_{s}=154 \mathrm{G}$. Typically, the conductivity and the Hall voltage were measured on $5 \times 10 \mathrm{~mm}^{2}$ samples with six gold contacts (without etching a Hall bar), using a d.c. current between $0.1 \mathrm{nA}$ and $1 \mathrm{~mA}$. For a $\mathrm{Zn}_{0.981} \mathrm{Mn}_{0.019} \mathrm{Te}: \mathrm{N}$ sample with the highest hole concentration the measurements were extended down to $100 \mathrm{mK}$ with the use of the lock-in technique. Magnetization studies were carried out down to $1.5 \mathrm{~K}$ employing either a superconducting quantum interference device (SQUID) set-up (with the magnetic field up to $1.3 \mathrm{kOe}$ ) or a vibrating sample magnetometer (with the field up to $20 \mathrm{kOe}$ ). In both cases the field was applied in the direction parallel to the film surface, so that no demagnetization effects had to be taken into account.

The values of hole and Mn concentrations were obtained from the studies of the Hall effect and magnetic susceptibility, respectively, according to procedures described in detail below. A hole concentration as high as $1.2 \times 10^{20} \mathrm{~cm}^{-3}$ was obtained in the case of $\mathrm{ZnTe}$ and $\mathrm{Zn}_{0.981} \mathrm{Mn}_{0.019}$ Te epilayers. ${ }^{5}$ To our knowledge, this is the largest hole concentration ever achieved for any II-VI semiconductor. However, the doping efficiency tends to decrease when the Mn content increases, either with or without additional $\mathrm{Zn}$ flux during the growth, so that $p \leqslant 10^{19} \mathrm{~cm}^{-3}$ for $x \geqslant 0.05$. The magnetic moment of a reference sample containing a ZnTe:N epilayer has been
TABLE I. Characteristics of the studied samples of $\mathrm{Zn}_{1-x} \mathrm{Mn}_{x}$ Te:N. Hole concentration $p$ was determined from Hall resistance, whereas effective Mn content $x_{\text {eff }}$ and the Curie-Weiss temperature $T_{\mathrm{CW}}$ from magnetic susceptibility at $4 \leqslant T \leqslant 20 \mathrm{~K}$, according to procedures described in Sec. III.

\begin{tabular}{rlc}
\hline \hline$p\left[\mathrm{~cm}^{-3}\right]$ & \multicolumn{1}{c}{$x_{\text {eff }}$} & $T_{\mathrm{CW}}[\mathrm{K}]$ \\
\hline $1.2 \times 10^{20}$ & 0 & 0 \\
$1.2 \times 10^{20}$ & 0.015 & 1.45 \\
$7 \times 10^{19}$ & 0.005 & \\
$3 \times 10^{19}$ & 0.025 & 2.3 \\
$1.5 \times 10^{19}$ & 0.027 & 2.4 \\
$9 \times 10^{18}$ & 0.0315 & 0.75 \\
$8 \times 10^{17}$ & 0.0285 & -0.4 \\
\hline \hline
\end{tabular}

measured with an applied magnetic field up $20 \mathrm{kG}$ and from room temperature down to $1.5 \mathrm{~K}$. A temperature independent, diamagnetic behavior was observed in this whole temperature range. We attribute this diamagnetic moment to the $800 \mu \mathrm{m}$-thick substrate (due to the thickness ratio, the contribution from the epilayer is three orders of magnitude smaller): It corresponds to a diamagnetic susceptibility (per unit volume) $\chi_{\text {substrate }}=-1.3 \times 10^{-6}$ e.m.u, in agreement with values known for bulk materials. We found no contribution from residual paramagnetic impurities in the substrate or in the $\mathrm{ZnTe}$ epilayers. In all samples with a $\mathrm{Zn}_{1-x} \mathrm{Mn}_{x} \mathrm{Te}$ epilayer, an additional, temperature dependent contribution could be observed below $80 \mathrm{~K}$, which we attribute to the $\mathrm{Mn}$ spins. Above $100 \mathrm{~K}$, this paramagnetic contribution becomes negligible with respect to the diamagnetic moment of the substrate (this is essentially due to the thickness ratio between the $\mathrm{Zn}_{1-x} \mathrm{Mn}_{x}$ Te layer and the substrate). Then, on each sample, the $\mathrm{Zn}_{1-x} \mathrm{Mn}_{x}$ Te contribution to the total magnetic moment was obtained from the measured signal, by subtracting the diamagnetic contribution from the substrate as determined on the same sample above $100 \mathrm{~K}$.

Table I presents the hole concentration $p$ as well as the effective Mn content $x_{\text {eff }}$ and Curie-Weiss temperature $T_{\mathrm{CW}}$, as determined for the studied samples according to procedures described in the next section.

\section{EXPERIMENTAL RESULTS}

\section{A. Conductance and magnetoresistance}

The binding energy of an effective mass acceptor in $\mathrm{ZnTe}$, evaluated from the Baldereschi-Lipari mode ${ }^{21}$ with the published values of the Luttinger parameters for $\mathrm{ZnTe},{ }^{22}$ is $59 \mathrm{meV}$. The same calculation gives the effective Bohr radius, $a^{*} \approx 1.3 \mathrm{~nm}$, which leads to the Mott critical density $N_{c}=\left(0.26 / a^{*}\right)^{3}=0.8 \times 10^{19} \mathrm{~cm}^{-3}$. Experimentally, substitutional nitrogen forms an even slightly shallower acceptor, with a binding energy as low as $53 \mathrm{meV} .{ }^{20} \mathrm{~A} \mathrm{ZnTe}$ sample with $p=1.2 \times 10^{20} \mathrm{~cm}^{-3}$ is clearly metallic, according to measurements down to pumped liquid helium temperature [Fig. 1(a)]. The conductivity $\sigma$ of a $\mathrm{Zn}_{1-x} \mathrm{Mn}_{x} \mathrm{Te}$ sample with $x=0.019$ and with the same hole density $(p=1.2$ $\times 10^{20} \mathrm{~cm}^{-3}$ ) is remarkably identical, if measured in a mag- 


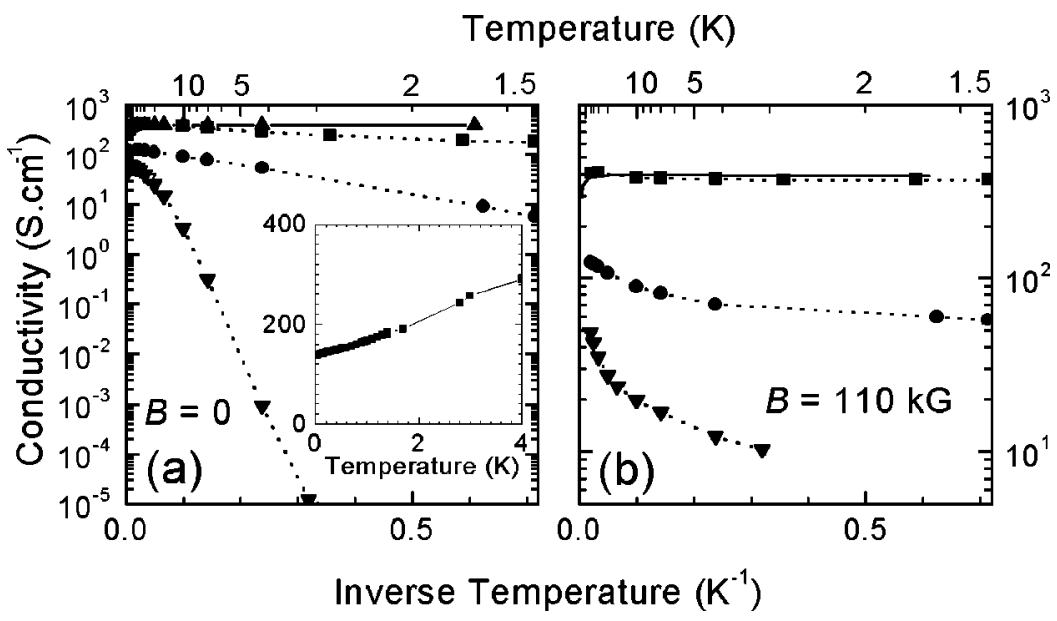

FIG. 1. Conductivity of $\mathrm{ZnTe}: \mathrm{N}$ and $\mathrm{Zn}_{1-x} \mathrm{Mn}_{x} \mathrm{Te}: \mathrm{N}$ as a function of the inverse temperature without (a) and with (b) an applied magnetic field of $110 \mathrm{kGs}$; up-triangles stand for $\mathrm{ZnTe}$ with $p=1.2 \times 10^{20} \mathrm{~cm}^{-3}$; other symbols are for $\mathrm{Zn}_{1-x} \mathrm{Mn}_{x} \mathrm{Te}$ with $x=0.019$ and $p$ $=1.2 \times 10^{20} \mathrm{~cm}^{-3}$ (squares); $x=0.005$ and $p=7 \times 10^{19} \mathrm{~cm}^{-3}$ (circles), and $x=0.038$ and $p=3 \times 10^{19} \mathrm{~cm}^{-3}$ (down triangles). The inset to (a) shows the temperature dependence of conductivity for $x=0.019$ and $p=1.2 \times 10^{20} \mathrm{~cm}^{-3}$ in zero magnetic field. The lines are drawn through experimental points; the solid line, drawn in both (a) and (b), represents the temperature dependence of resistivity in the metallic ZnTe sample in zero magnetic field. The data point to temperature dependent localization by magnetic disorder, an effect suppressed by a high magnetic field.

netic field, as shown in Fig. 1(b), where data taken in 110 kOe are summarized. The same plot illustrates how the conductivity decreases when the hole concentration diminishes, and demonstrates that the temperature dependence of the conductivity remains weak even for the less doped sample (with $p=3 \times 10^{19} \mathrm{~cm}^{-3}$ ). In particular, the conductivity of all samples in the magnetic field stay higher or of the order of the Mott minimum metallic conductivity $\sigma_{\min }$ $\approx 0.03 N_{c}^{1 / 3} e^{2} / \hbar \approx 15 \mathrm{Scm}^{-1}$.

The situation is very different without the applied field [Fig. 1(a)]. The sample with the highest doping level exhibits a small decrease of conductivity-by a factor of 2.5 between 10 and $0.1 \mathrm{~K}$, as shown in the inset to Fig. 1(a). Thus, this sample has to be considered as metallic, also in the absence of the magnetic field, as its conductivity remains an order of magnitude greater than $\sigma_{\mathrm{min}}$, even at $100 \mathrm{mK}$. In contrast, the conductivity of samples with slightly smaller hole concentrations decreases rather dramatically when lowering the temperature indicating that the holes become localized at low temperatures in the absence of the field. Such localization induced by magnetic disorder was also detected in Bridgman-grown $\mathrm{Zn}_{1-x} \mathrm{Mn}_{x}$ Te:P with $p<10^{19} \mathrm{~cm}^{-3} \cdot{ }^{23} \mathrm{We}$ have checked that no reentrant metallic behavior occurs in such samples down to $100 \mathrm{mK}$. In contrast, a comparison of Figs. 1(a) and 1(b) points to the presence of a field-induced insulator-to-metal transition, and indeed a colossal (several orders of magnitude) negative magnetoresistance is observed at low temperature. Additionally, a weak positive magnetoresistance in the low field range is also visible, as reported elsewhere. $^{24}$

The above effects, the temperature dependent localization as well as the positive and negative magnetoresistances, are qualitatively similar to those observed previously for $n$-type DMS. ${ }^{9,25,26}$ In particular, the positive magnetoresistance results from the giant Zeeman splitting of band states in DMS, which modifies quantum corrections to the conductivity at the localization boundary. ${ }^{25}$ Under the same conditions, a strong spin dependent scattering of itinerant carriers by ferromagnetic spin puddles [bound magnetic polarons (BMP)] is thought ${ }^{25,27}$ to account for the shifting of the metalinsulator transition (MIT) towards higher impurity concentrations as well as for the rapid increase of the resistivity when decreasing the temperature, and the associated unusually strong negative magnetoresistance. The latter is enhanced by the increase of the carrier kinetic energy, which results from their redistribution between the spin subbands, an effect particularly important in $p$-type DMS. ${ }^{9,28}$

In conclusion, the studies of conductance as a function of temperature and magnetic field reveal qualitatively similar properties of $n$-type and $p$-type II-VI DMS in the vicinity of the MIT. In particular, the temperature dependent localization and negative magnetoresistance indicate that the efficiency of spin disorder scattering increases at low temperature. This means that ferromagnetic correlation grows when the temperature decreases. However, the correlation length of some ferromagnetic puddles has to remain small, of the order of the de Broglie wavelength of the itinerant holes, to result in efficient scattering of the hole spins.

\section{B. Hall resistivity}

Figure 2 shows the Hall resistivity $\rho_{x y}$ measured at various temperatures for the highly doped $\mathrm{Zn}_{0.981} \mathrm{Mn}_{0.019} \mathrm{Te}$ sample. The quoted hole concentration is deduced from the slope of the room temperature Hall resistance. We found that $\rho_{x y}$ is linear in the magnetic field and temperature independent down to $150 \mathrm{~K}$. In the case of the ZnTe sample, this normal Hall effect $\rho_{x y}$ linear in the field $H$ and temperature independent, is observed down to $1.6 \mathrm{~K}$ (not shown). By contrast, in the case of $\mathrm{Zn}_{1-x} \mathrm{Mn}_{x} \mathrm{Te}$, when decreasing the temperature below $100 \mathrm{~K}$, one observes first an increase of the slope of the Hall resistance, and then a strong nonlinear- 


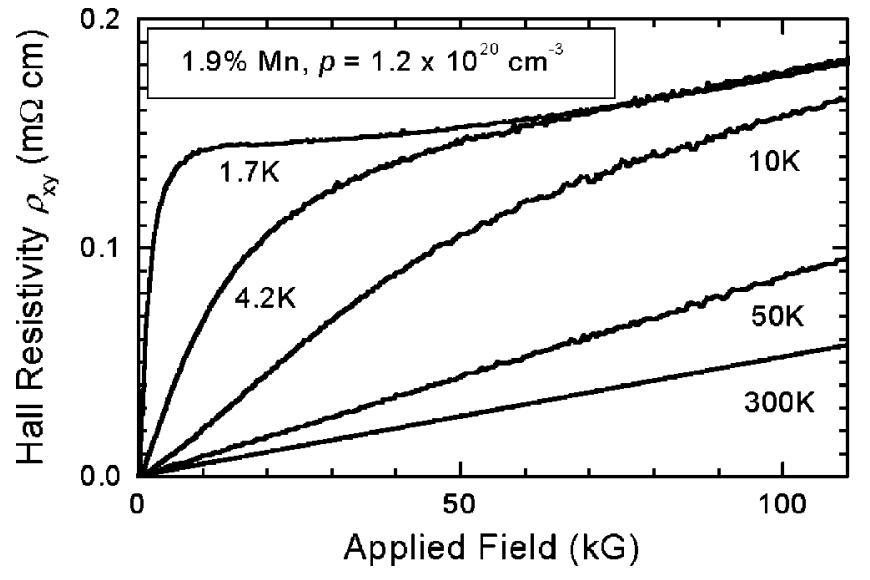

FIG. 2. Hall resistivity versus magnetic field at different temperatures, from room temperature down to $1.7 \mathrm{~K}$ in metallic $p-\mathrm{Zn}_{0.981} \mathrm{Mn}_{0.019} \mathrm{Te}: \mathrm{N}$. The nonlinear temperature dependent component is assigned to the extraordinary Hall effect, which strongly increases on approaching the ferromagnetic phase transition (see Fig. 4).

ity. This "extraordinary" (or "'anomalous") spin-dependent Hall effect $\rho_{x y}^{(a n)}$ has already been discussed in Ref. 24. It is clearly observed in III-V DMS, ${ }^{2}$ but not in $n$-doped II-VI DMS. ${ }^{26}$ Its large magnitude stems from the importance of the spin-orbit coupling in the valence band and from the large polarization of the hole liquid. The latter results from the giant Zeeman splitting of the four hole subbands, which is proportional to the magnetization of the Mn spins. At low temperature and high field, the $\mathrm{Mn}$ or the hole spin polarization saturates, and then the Hall resistivity exhibits again a linear dependence on the applied field, with the same slope as at room temperature. Thus, while the spin-dependent component is too large to allow us to determine the hole density at low temperatures and in small fields, its magnitude becomes negligibly small at room temperature, or at lowtemperature in high fields. For these two cases, the slope of the Hall resistance was found to be identical, giving unambiguously the value of the hole density.

In the case of less doped samples, we could measure the Hall resistivity down to typically $10 \mathrm{~K}$, with the same conclusions, i.e., (i) the normal Hall effect dominates at temperatures above $150 \mathrm{~K}$; (ii) the Hall resistivity varies linearly with the magnetic field at low temperature in sufficiently large magnetic fields, and (iii) a strong spin-dependent component appears at weak magnetic fields and at low tempera- ture, though its accurate determination in this region is hampered by the large value of the resistance and a strong magnetoresistance. As mentioned above, the Hall resistance provides direct information on the degree of spin polarization $\mathcal{P}$ of the carrier liquid. However, depending on the dominant mechanism leading to the extraordinary Hall effect, ${ }^{29} \mathcal{P}$ is proportional either to the Hall angle, $\mathcal{P} \sim\left(\rho_{x y}\right.$ $\left.-\rho_{x y}^{(o)}\right) / \rho_{x x}$ ("skew scattering" mechanism) or to the offdiagonal conductivity component, $\mathcal{P} \sim\left(\rho_{x y}-\rho_{x y}^{(o)}\right) / \rho_{x x}^{2}$ ("side jump" effect). Accordingly, the form of $\mathcal{P}(T, B)$ deduced from the Hall and resistivity measurements depends on the assumed model. The work aiming in elucidating the actual origin of the extraordinary Hall effect in $p$-type DMS is under way.

\section{Magnetic properties}

\section{Antiferromagnetic superexchange}

The magnetic properties of undoped $\mathrm{Zn}_{1-x} \mathrm{Mn}_{x}$ Te layers and bulk crystals are well known and the magnetization $M$ in the magnetic field $H$ can be described by a modified Brillouin function $B_{S}, 9,30$

$$
M=S g \mu_{B} N_{0} x_{\mathrm{eff}} B_{S}\left[\frac{S g \mu_{B} H}{k_{B}\left(T+T_{\mathrm{AF}}\right)}\right] .
$$

The corresponding low-field susceptibility is given by

$$
\begin{gathered}
\chi_{\mathrm{Mn}}=C_{0} x_{\mathrm{eff}} /\left(T+T_{\mathrm{AF}}\right), \\
C_{0}=S(S+1) g_{\mathrm{Mn}}^{2} \mu_{B}^{2} N_{0} / 3 k_{B},
\end{gathered}
$$

where the Mn spin $S=5 / 2$ and the Landé factor $g_{\mathrm{Mn}}=2.0$; the density of cation sites in ZnTe is $N_{0}=1.76 \times 10^{22} \mathrm{~cm}^{-3}$. The two empirical parameters $x_{\mathrm{eff}}<x$ and $T_{\mathrm{AF}}>0$ take into account the antiferromagnetic superexchange interaction between the Mn spins. At temperatures $T<20 \mathrm{~K}$, the effective density of spin $x_{\text {eff }} N_{0}$ is smaller than the density of Mn ions $x N_{0}$ since the nearest-neighbor (n.n.) Mn pairs are blocked antiparallel due to their strong superexchange interaction $J_{1} / k_{B} \cdot{ }^{9}$ Only "free spins,', which are not involved in these n.n. pairs, contribute to $x_{\text {eff }}$. The phenomenological CurieWeiss temperature $T_{\mathrm{AF}}$ describes the effect of antiferromagnetic interactions between more distant Mn pairs. Note that these two parameters $x_{\mathrm{eff}}$ and $T_{\mathrm{AF}}$ are valid over a field and temperature range (low field and $T<20 \mathrm{~K}$ ) which is relevant for the present study. Particularly, $T_{\mathrm{AF}}$ should not be con-
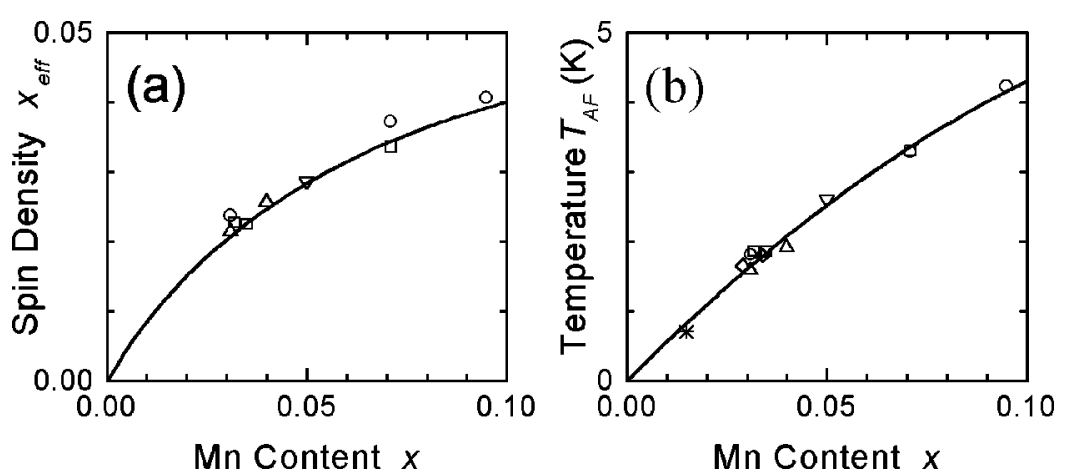

FIG. 3. Empirical values of parameters characterizing antiferromagnetic interactions in undoped $\mathrm{Zn}_{1-x} \mathrm{Mn}_{x}$ Te: (a) effective content $x_{\text {eff }}$ of unpaired $\mathrm{Mn}$ spins and (b) antiferromagnetic Curie-Weiss temperature $T_{\mathrm{AF}}$ determined by interactions between non-nearest-neighbors spins. Lines present fits of Eqs. (4) and (5) to experimental data (symbols) taken from Ref. 31 (squares: Barilero et al.; down-triangles: Lascaray et al.; up-triangles: Shapira et al.; circles: Twardowski et al.; star: present study, magnetooptical spectroscopy for an undoped sample). 


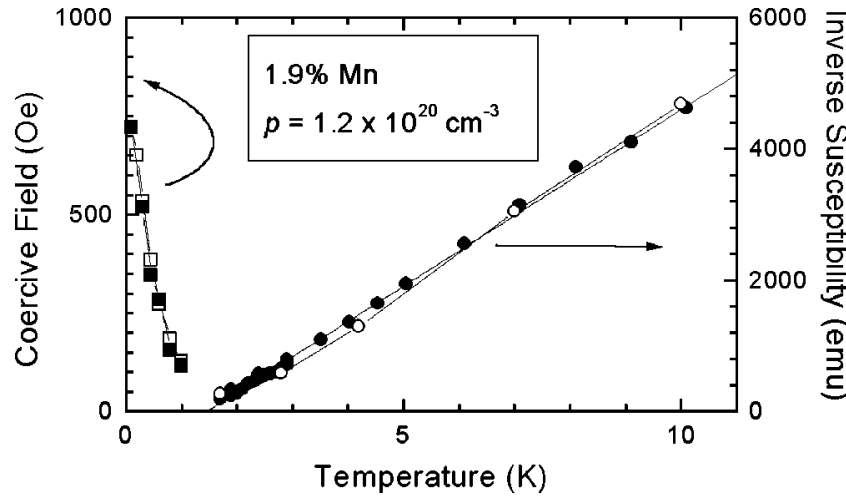

FIG. 4. Inverse magnetic susceptibility (per unit volume) vs. temperature for metallic $p-\mathrm{Zn}_{0.981} \mathrm{Mn}_{0.019}$ Te (full circles). Solid line shows the linear fit, which serves us to determine the effective Mn content $x_{\text {eff }}$ and the Curie-Weiss temperature $T_{\mathrm{CW}}$ displayed in Table I. The inverse Hall resistivity (open circles) and the halfwidth of hysteresis loops as determined from Hall resistivity (full squares) and longitudinal resistivity (open squares) are shown for the same sample. These data point to a ferromagnetic phase transition in the vicinity of $1.4 \mathrm{~K}$.

fused with the regular Curie-Weiss temperature determined in the high temperature range. Fitting a set of data from the literature $^{31}$ leads us to use the same purely phenomenological expressions as already proposed for $\mathrm{Cd}_{1-x} \mathrm{Mn}_{x} \mathrm{Te}^{32}$ (Fig. 3):

$$
\begin{gathered}
x_{\text {eff }}=x\left(0.26 e^{-43.3 x}+0.73 e^{-6.2 x}+0.01\right), \\
T_{\mathrm{AF}}[\mathrm{K}]=58 x-150 x^{2} .
\end{gathered}
$$

The variation of $x_{\text {eff }}$ is described by the same parameters as in $\mathrm{Cd}_{1-x} \mathrm{Mn}_{x} \mathrm{Te},{ }^{32}$ which comes as no surprise since a statistical evaluation of concentration of unpaired spins explains satisfactorily the experimental findings. ${ }^{33,34}$

\section{Metallic regime-Ferromagnetic phase transition}

Experimental results demonstrating the presence of the hole-induced ferromagnetism in the metallic $\mathrm{Zn}_{0.981} \mathrm{Mn}_{0.019}$ Te:N sample are summarized in Figs. 4 and 5. The susceptibility is determined from the value of magneti- zation at $1 \mathrm{kOe}$ above $5 \mathrm{~K}$, and from the Arrott plots $\left(M^{2}\right.$ vs. $H / M$ plots) at lower temperatures, for which the curvature of the Brillouin function becomes significant. The inverse magnetic susceptibility plotted as a function of temperature (Fig. 4) gives the value of the Curie constant $C_{0}$, which corresponds to $x_{\text {eff }}=0.015$. According to Eq. (4) this leads to $x$ $=0.019$. The data collected in Fig. 3(b) imply that for such a Mn concentration the antiferromagnetic superexchange results in the Curie-Weiss temperature $T_{\mathrm{CW}}=-1.0 \mathrm{~K}$, which would be observed for undoped samples. In contrast, the susceptibility and Hall resistance data for the metallic $\mathrm{Zn}_{0.981} \mathrm{Mn}_{0.019} \mathrm{Te}: \mathrm{N}$ sample as depicted in Fig. 4, point to a positive value of the Curie-Weiss temperature $T_{\mathrm{CW}}=1.45$ $\pm 0.1 \mathrm{~K}$. This demonstrates that the itinerant holes mediate ferromagnetic interactions among the Mn spins, which overcompensate the antiferromagnetic superexchange. However, the small value of the resulting Curie-Weiss temperature indicates that the ferromagnetic and antiferromagnetic interactions are of similar magnitudes. The competition between them constitutes an important ingredient of the carrierinduced ferromagnetism in II-VI semiconductors.

In order to probe the magnetic ordering below $T_{\mathrm{CW}}$, the measurements of the diagonal and Hall resistivities have been extended down to $100 \mathrm{mK}$. As shown in Fig. 5, clearly visible hystereses develop in both $\rho_{x x}$ and $\rho_{x y}$ on lowering the temperature. This indicates that the low temperature phase is ferromagnetic. At the same time, the temperature dependence of hysteresis widths presented in Fig. 4, points to the Curie temperature $T_{C}$ that is only slightly lower than $T_{\mathrm{CW}}$. The latter means that the mean-field approximation constitutes a good starting point for the description of the ferromagnetism in the studied system, an expected finding in view of the long-range character of the carrier-mediated exchange interaction. ${ }^{35}$

\section{Ferromagnetic interactions mediated by localized holes}

Since the doping efficiency decreases and the magnetic disorder increases with the Mn concentration, the holes are localized in our samples with $x>0.03$. Actually, the conductance at $T<4 \mathrm{~K}$ drops by several orders of magnitude on crossing the MIT. For instance, according to Fig. 1, zero-

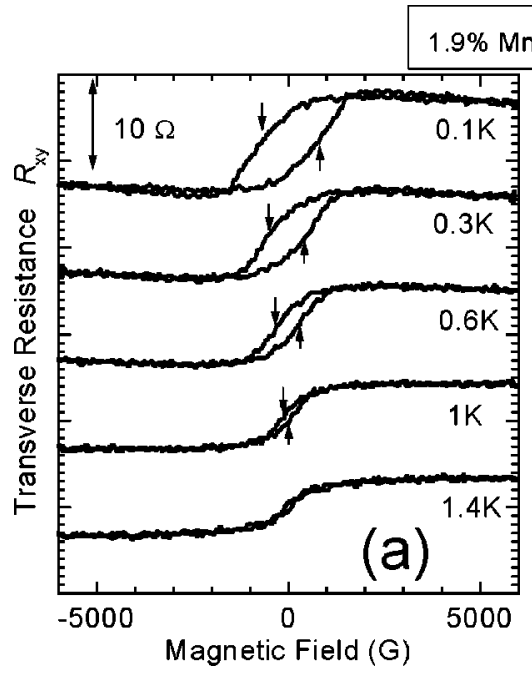

Magnetic Field (G)

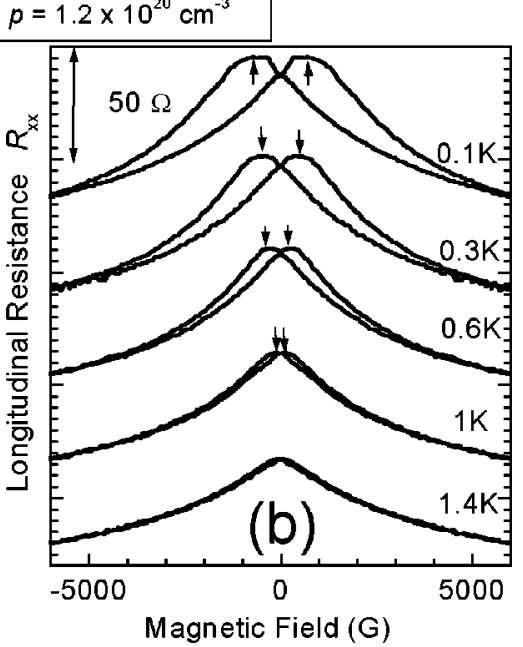

$085201-5$
FIG. 5. Low temperature magnetoresistance (a) and Hall resistance (b) for metallic $p-\mathrm{Zn}_{0.981} \mathrm{Mn}_{0.019}$ Te. The vertical lines mark the width of the hysteresis loops, which is depicted as a function of temperature in Fig. 4. 

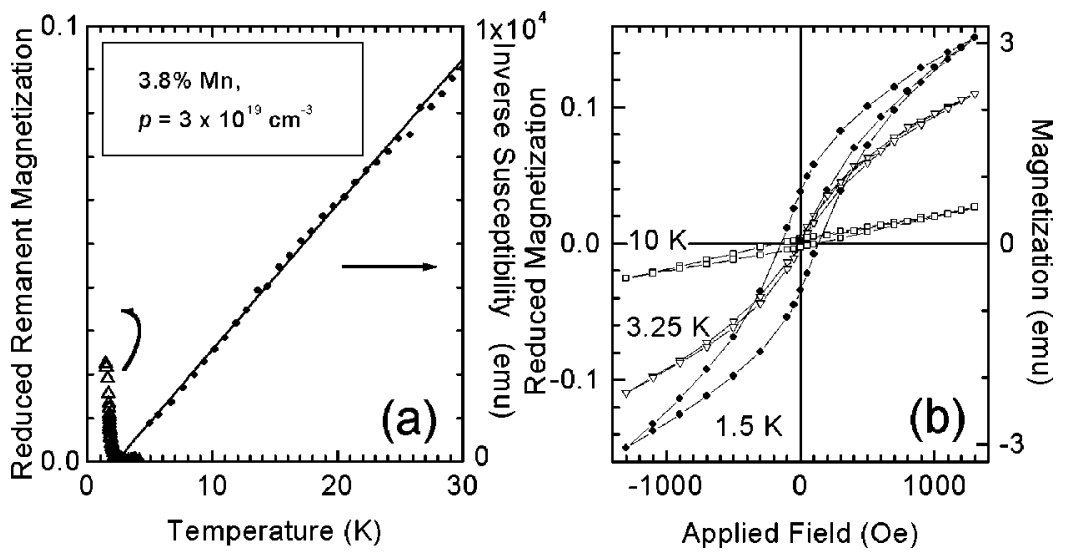

FIG. 6. (a) Inverse magnetic susceptibility (circles) and remanent magnetization (triangles) after magnetization cycling up to $1.3 \mathrm{kOe}$ for $p-\mathrm{Zn}_{0.962} \mathrm{Mn}_{0.038} \mathrm{Te}$ on the insulator side of the metal-to-insulator transition. Solid line shows the linear fit, which serves us to determine the effective Mn content $x_{\text {eff }}$ and the Curie-Weiss temperature $T_{\mathrm{CW}}$ summarized in Table I. (b) Magnetization cycles at various temperatures displayed in the units of the saturation value. field conductance of $\mathrm{Zn}_{0.962} \mathrm{Mn}_{0.038} \mathrm{Te}: \mathrm{N}$ with $p=3 \times 10^{19}$ $\mathrm{cm}^{-3}$ is smaller by more than five orders of magnitude than that of $\mathrm{Zn}_{0.981} \mathrm{Mn}_{0.019}$ Te: $\mathrm{N}$ with $p=1.2 \times 10^{20} \mathrm{~cm}^{-3}$. Thus, an interesting question arises how this dramatic difference in transport properties will affect the hole-mediated ferromagnetic interactions.

Figure 6(a) shows the temperature dependence of the inverse magnetic susceptibility of $\mathrm{Zn}_{0.962} \mathrm{Mn}_{0.038}$ Te: $\mathrm{N}$ with $p$ $=3 \times 10^{19} \mathrm{~cm}^{-3}$. Again the susceptibility was determined from the magnetization at $1 \mathrm{kOe}$ at higher temperatures and from the Arrott plots at lower temperatures. A clear dopinginduced positive shift of the Curie-Weiss temperature $T_{\mathrm{CW}}$ is put into evidence: we observe $T_{\mathrm{CW}}=2.3 \mathrm{~K}$ for a sample with $x=0.038\left(x_{\text {eff }}=0.025\right)$ and $p=3 \times 10^{19} \mathrm{~cm}^{-3}$, instead of $T_{\mathrm{CW}}=-T_{\mathrm{AF}}=-2 \mathrm{~K}$, which-according to Fig. 3(b)-is expected for an undoped sample with the same Mn content.

Figure 6(b) shows magnetization loops measured on the sample in question. A paramagnetic behavior is observed above $T_{\mathrm{CW}}$, with a linear dependence of the magnetization on the magnetic field at high temperature (e.g., at $10 \mathrm{~K}$ ), and an onset of the field-induced saturation of magnetization at lower temperature (e.g., at $3.2 \mathrm{~K}$ ). Magnetization cycles measured at still lower temperature (e.g., at $1.5 \mathrm{~K}$ ) exhibit hysteresis: The remanent magnetization is displayed in Fig. 6(a). A slowly decaying component ( $20 \%$ of the total signal at the lowest temperature) was observed after shutting down the field: The values of remanent magnetization plotted in Fig. 6(a) were measured after $15 \mathrm{~min}$, which corresponded to a complete decay of the slowly varying component. We may note that the magnitude of remanent magnetization remains rather small compared to the total amount of Mn spins deduced from the susceptibility in the paramagnetic phase. This is only partly due to the fact that the applied field remains low and these are probably only minor cycles. Such a small magnitude may indicate also that the easy axis is out of the plane. However, effects due to magnetic disorder (competition of ferromagnetic and antiferromagnetic interactions) as well as due to electrostatic disorder (mesoscopic fluctuations in the density-of-states) might also be present and drive the system to a spin-glass phase or to a ferromagnetic phase with atypically long relaxation times. ${ }^{17}$

Figure 7 shows the temperature dependence of the inverse magnetic susceptibility for two $\mathrm{Zn}_{1-x} \mathrm{Mn}_{x}$ Te layers with an approximate Mn content $x \approx 0.04$ and even smaller hole con- centrations than those in the samples discussed above. Again, doping-induced positive shift of the Curie-Weiss temperature $T_{\mathrm{CW}}$ is clearly visible, as instead of $T_{\mathrm{CW}}=-T_{\mathrm{AF}}=-2.3 \mathrm{~K}$ expected for $x=0.04$, the observed values of $T_{\mathrm{CW}}$ are 2.4 and $-0.4 \mathrm{~K}$ for $p=1.5 \times 10^{19} \mathrm{~cm}^{-3}$ and $p=7 \times 10^{17} \mathrm{~cm}^{-3}$, respectively.

We conclude that the Curie-Weiss temperature does not exhibit any critical behavior on crossing the MIT. This demonstrates the existence of ferromagnetic interactions of a similar magnitude on both sides of the MIT. At the same time, it is possible that a state with an exotic space or time spin correlation, more complex than that of a simple collinear ferromagnet, develops in the insulator phase.

\section{MODEL AND MATERIAL PARAMETERS}

\section{A. Zener model and spin susceptibility of carriers}

As discussed above, our study provides the values of Curie-Weiss $T_{\mathrm{CW}}$ as a function of the effective spin density $x_{\text {eff }}$, deduced from the Curie constant, and the hole density $p$,

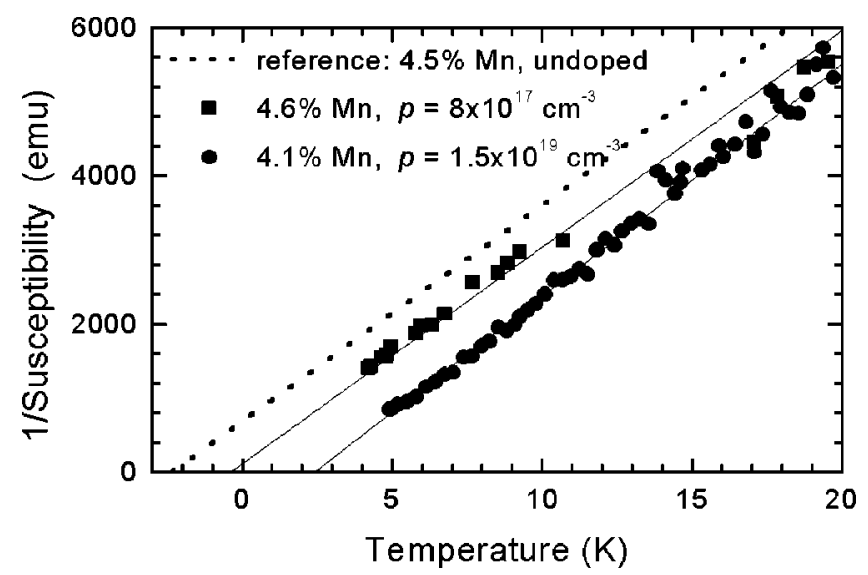

FIG. 7. Inverse magnetic susceptibility (squares) for two $p-\mathrm{Zn}_{1-x} \mathrm{Mn}_{x}$ Te samples with similar Mn content $x \approx 0.045$ but different hole concentrations, both on the insulator side of the metalto-insulator transition. Solid lines show linear fit, which serves us to determine the effective Mn content $x_{\text {eff }}$ and the Curie-Weiss temperature $T_{\mathrm{CW}}$ displayed in Table I. The dotted line presents the dependence expected for an undoped sample with a similar Mn content. 
determined from the Hall effect measurements. Knowing $x_{\text {eff }}$, we determine from Eqs. (4) and (5) the actual Mn content $x$ and $T_{\mathrm{AF}}$. The latter would be observed for undoped samples with the same composition $x$, and characterizes the strength of antiferromagnetic interactions between Mn spins more distant than the nearest neighbor pairs. In DMS, the coupling between the hole of spin $\mathbf{s}$ and position $\mathbf{r}$, and the Mn spin $\mathbf{S}_{i}$ localized at $\mathbf{R}_{i}$, is well described by a local exchange interaction ${ }^{9,30,36}$

$$
H_{p-d}=-\beta s \cdot \mathbf{S}_{i} \delta\left(r-\mathbf{R}_{i}\right),
$$

where the spin-hole exchange energy is known from magneto-spectroscopy to be $\beta N_{0}=-1.1 \mathrm{eV}$ in $\mathrm{Zn}_{1-x} \mathrm{Mn}_{x}$ Te. ${ }^{37}$ This interaction can be used to evaluate the energy $J_{i j}$ of the carrier-mediated RKKY exchange coupling between the Mn spins $i$ and $j$. This coupling exhibits the well-known oscillations as a function of the distance between the Mn pairs with the period determined by the Fermi wave vector $k_{\mathrm{F}}^{-1}$. Then, in order to obtain the mean-field value of $T_{\mathrm{CW}}$, the interaction energy has to be averaged over the distribution of the Mn spins. This will be elaborated later. Now we consider the limit of low carrier density, in which the mean Mn-Mn distance is small with respect to $k_{\mathrm{F}}^{-1}$. In such a case, the continuous-medium limit of the RKKY model, i.e., the Zener model, ${ }^{38}$ can be used. ${ }^{3,14,39}$ The meanfield version of this model can be summarized as follows.

First we use the molecular-field and virtual-crystal approximations to relate the spin polarization of the hole liquid to the Mn magnetization $\mathbf{M}$. The result is

$$
\left\langle s_{z}\right\rangle=\beta \widetilde{\chi}_{h} \sum_{i}\left\langle S_{z}^{i}\right\rangle \delta\left(\mathbf{r}-\mathbf{R}_{i}\right)=\beta \widetilde{\chi}_{h} x_{\mathrm{eff}} N_{0}\left\langle S_{z}\right\rangle,
$$

where the first-line summation runs over a discrete distribution of the Mn spins, and is replaced in the second line by an average spin projection on the direction of $\mathbf{M}$, taken here as $z$ axis. The quantity $\tilde{\chi}_{h}$ is the carrier spin susceptibility, which in the absence of the spin-orbit interaction would correspond to the Pauli magnetic susceptibility with the $g \mu_{B}$ factor omitted.

We also adopt the mean-field approximation (MFA) to obtain $M$

$$
M / g_{\mathrm{Mn}} \mu_{B}=-x_{\mathrm{eff}} N_{0}\left\langle S_{z}\right\rangle=\widetilde{\chi}_{\mathrm{Mn}}\left(g_{\mathrm{Mn}} \mu_{B} H-\beta\left\langle s_{z}\right\rangle\right),
$$

where

$$
\widetilde{\chi}_{\mathrm{Mn}}=\frac{\widetilde{C}_{0} x_{\mathrm{eff}}}{T+T_{\mathrm{AF}}} \text { and } \widetilde{C}_{0}=\frac{S(S+1) N_{0}}{3 k_{B}} .
$$

Combining Eqs. (7) and (8) we are led to ${ }^{3}$

$$
M=\frac{\chi_{\mathrm{Mn}}}{1-\widetilde{\chi}_{\mathrm{Mn}} \widetilde{\chi}_{h} \beta^{2}} \quad H=\frac{C_{0} x_{\mathrm{eff}}}{T-T_{\mathrm{CW}}} H,
$$

with

$$
T_{\mathrm{CW}}=T_{\mathrm{F}}-T_{\mathrm{AF}} \text { and } T_{\mathrm{F}}=x_{\mathrm{eff}} \widetilde{C}_{0} \beta^{2} \widetilde{\chi}_{h}
$$

where, within the MFA, $T_{\mathrm{CW}}$ is equal to the Curie temperature $T_{C}$. Usually, however, the MFA, which can also be regarded as a high temperature expansion, gives a better estimate of $T_{\mathrm{CW}}$ than of $T_{C}$.

The above reasoning can easily be generalized to the case of a phase transition to a spatially modulated ground-state, characterized by nonzero magnetization $\mathbf{M}(\mathbf{q})$. The corresponding mean-field value of the ordering temperature $T_{C}(\mathbf{q})$ is given by the solution of the equation ${ }^{40}$

$$
\widetilde{\chi}_{\mathrm{Mn}}(\mathbf{q}, T) \widetilde{\chi}_{h}(\mathbf{q}, T) \beta^{2}=1 .
$$

In the case of the conduction band, the periodic part of the Bloch wave function is $S$-like, so that spin dynamics is not perturbed by the spin-orbit interaction. The spin susceptibility of a degenerate carrier liquid at $q=0$ is then directly related to the density-of-states at the Fermi energy,

$$
\widetilde{\chi}_{e}=\frac{1}{4} A_{\mathrm{F}} \rho\left(E_{\mathrm{F}}\right) .
$$

Here, the prefactor $A_{\mathrm{F}}$ is the Fermi liquid parameter that takes into account the enhancement of the spin susceptibility by the carrier-carrier exchange interactions. However, in the case of the holes involving $P$-like states, the influence of the spin-orbit coupling has to be taken into account. ${ }^{3,14}$

\section{B. Spin susceptibility of holes}

The valence band in ZnTe is characterized by a rather strong spin-orbit coupling, $\Delta_{0}=0.96 \mathrm{eV} .{ }^{41}$ Hence at the center of the Brillouin zone the $\Gamma_{7}$ split-off band is well separated from the $\Gamma_{8}$ quadruplet, and for small wave vectors, the dispersion can be calculated by using the $4 \times 4$ Luttinger Hamiltonian. We adopt the experimental values of the Luttinger parameters ${ }^{22} \gamma_{1}=3.8, \gamma_{2}=0.72$, and $\gamma_{3}=1.3$, and use the spherical approximation, ${ }^{21}$ i.e., we replace $\gamma_{2}$ and $\gamma_{3}$ by $\gamma_{s}=\frac{2}{5} \gamma_{2}+\frac{3}{5} \gamma_{3}$. In this approximation, the values of the heavy-hole mass $m_{\mathrm{hh}}=0.60 m_{0}$ and the light-hole mass $m_{\mathrm{lh}}$ $=0.17 m_{0}$, are independent of the direction of the wave vector $\mathbf{k}$.

We are interested in the response function of the holes for the molecular field applied along the $z$ direction. However, at $k \neq 0$, the periodic parts of the Bloch functions are the eigenstates of $\mathbf{J} \cdot \mathbf{k} / k$, i.e., the quantization axis is along $\mathbf{k}$. The corresponding eigenfunctions, $| \pm 3 / 2, \mathbf{k}\rangle$ for the heavy holes and $| \pm 1 / 2, \mathbf{k}\rangle$ for the light holes, can be obtained from the eigenfunctions of $J_{z},| \pm 3 / 2\rangle_{z}$ and $| \pm 1 / 2\rangle_{z}$, by applying the corresponding rotation matrix within the $\Gamma_{8}$ quadruplet, identical to the rotation matrix within a $J=3 / 2$ quadruplet. The important point here is that $s_{z}$ in the basis of the eigenfunctions $| \pm 3 / 2, \mathbf{k}\rangle$ and $| \pm 1 / 2, \mathbf{k}\rangle$ reads 


$$
s_{z}=\left[\begin{array}{ccrc}
\frac{\cos \Theta}{2} & 0 & -\frac{\sin \Theta}{2 \sqrt{3}} & 0 \\
0 & -\frac{\cos \Theta}{2} & 0 & -\frac{\sin \Theta}{2 \sqrt{3}} \\
-\frac{\sin \Theta}{2 \sqrt{3}} & 0 & \frac{\cos \Theta}{6} & -\frac{\sin \Theta}{3} \\
0 & -\frac{\sin \Theta}{2 \sqrt{3}} & -\frac{\sin \Theta}{3} & -\frac{\cos \Theta}{6}
\end{array}\right],
$$

where $\Theta$ is the polar angle of the wave vector $\mathbf{k}$. There are no matrix elements between states with different $\mathbf{k}$ vectors.

The longitudinal component of the hole spin susceptibility is

$$
\widetilde{\chi}_{h}(\mathbf{q})=2 \sum_{i, j, \mathbf{k}} \frac{\left|\left\langle i, \mathbf{k}\left|s_{z}\right| j, \mathbf{k}+\mathbf{q}\right\rangle\right|^{2}}{E_{j, \mathbf{k}+\mathbf{q}}-E_{i, \mathbf{k}}} f\left(E_{i, \mathbf{k}}\right)\left[1-f\left(E_{j, \mathbf{k}+\mathbf{q}}\right)\right],
$$

where $|i, \mathbf{k}\rangle$ are the periodic part of the Bloch functions, $E_{i, \mathbf{k}}=\hbar^{2} k^{2} / 2 m_{\mathrm{hh}}$ or $\hbar^{2} k^{2} / 2 m_{\mathrm{lh}}$, and $f\left(E_{i, \mathbf{k}}\right)$ is the FermiDirac distribution function for the corresponding hole subbands. The RKKY interaction energy is proportional to the Fourier transform of Eq. (15). In the framework of the Zener model of ferromagnetism, we are interested in $\widetilde{\chi}_{h}(\mathbf{q}=0)$. The result that is obtained after a straightforward calculation for the degenerate hole gas assumes the form

$$
\tilde{\chi}_{h}=\frac{1}{4} A_{\mathrm{F}} \rho\left(E_{\mathrm{F}}\right)\left[\frac{1}{3}+\frac{8}{9} \frac{m_{\mathrm{hh}}^{3 / 2} m_{\mathrm{lh}}-m_{\mathrm{lh}}^{3 / 2} m_{\mathrm{hh}}}{\left(m_{\mathrm{hh}}-m_{\mathrm{lh}}\right)\left(m_{\mathrm{hh}}^{3 / 2}+m_{\mathrm{lh}}^{3 / 2}\right)}\right],
$$

where the Fermi liquid parameter $A_{\mathrm{F}}$, to be discussed later, represents the effect of the hole-hole interaction, and

$$
\rho\left(E_{\mathrm{F}}\right)=\left(m_{\mathrm{hh}}^{3 / 2}+m_{\mathrm{lh}}^{3 / 2}\right)^{2 / 3}\left(3 \pi^{2} p\right)^{1 / 3} / \pi^{2} \hbar^{2} .
$$

The two terms in the square brackets in Eq. (16) describes two distinct effects of the spin-orbit interaction on the hole spin susceptibility:

- the first arises from terms which are diagonal within the heavy-hole and within the light-hole subbands. Thus, the spin-orbit interaction reduces intra-band hole spin polarization by a factor of three. Another, more intuitive, description is obtained by noting that applying the molecular field $B$ along the $z$ direction splits the Fermi surface into two (up and down) surfaces, with the Zeeman splitting of the heavy holes proportional to $B\left\langle \pm 3 / 2, \mathbf{k}\left|s_{z}\right| \pm 3 / 2, \mathbf{k}\right\rangle$ $= \pm B \cos \Theta,{ }^{36}$ as shown in Fig. 8. The spin polarization is obtained by integrating $s_{z}$ over the states between the two Fermi surfaces, both $s_{z}$ and the population depending on $\Theta$. This means, in particular, that the spin polarization of the holes, i.e., the spin component of their magnetization, is not simply proportional to the difference in the up and down populations, as it would be the case for the electrons in the conduction band. By integrating the sum of the square of the relevant matrix elements over the solid angle

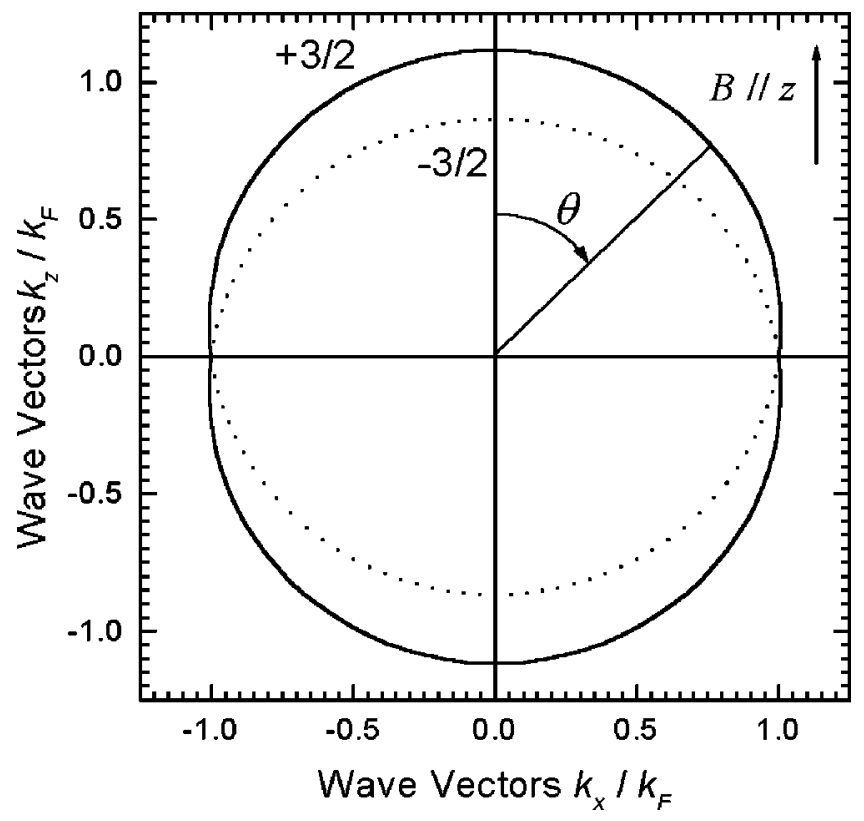

FIG. 8. Cross section of the heavy hole Fermi surface in presence of the exchange field (magnetization) applied along $z$ direction. The plot is shown for $\Sigma_{z}(B) / E_{\mathrm{F}}=0.5$, where $\Sigma_{z}(B)$ is the spin-splitting at $k=0$, but the calculation of the spin susceptibility is performed in the limit of vanishing $\Sigma_{z}(B) / E_{\mathrm{F}}$.

we obtain the reduction factor $\int_{0}^{\pi} \cos ^{2} \Theta d \cos \Theta / \int_{0}^{\pi} d \cos \Theta=1 / 3$. Hence, this reduction factor expresses the fact that the heavy-hole spin is quantized along the direction of $\mathbf{k}$, so that exchange splitting vanishes for $\mathbf{k} \perp \mathbf{B}$. A similar procedure for the light-hole subband leads to the same reduction factor $1 / 3$.

- The second term arises from nondiagonal terms coupling the heavy and light-hole subbands, that is, it represents interband spin polarization. Then, the Fermi distribution functions define the range of integration, from the Fermi wave vector of the light-holes to that of the heavy-holes. The corresponding contribution to $T_{\mathrm{CW}}$ can be viewed as the manifestation of the Bloembergen-Rowland mechanism ${ }^{9}$ of the indirect spin-spin exchange interaction, allowed here by the spin-orbit coupling.

Introducing into Eq. (16) the values of the hole effective masses corresponding to $\mathrm{ZnTe}$ we find that the spin-orbit interaction reduces the hole spin susceptibility by a factor of 2.1,

$$
\tilde{\chi}_{h}=\frac{1}{4} \rho\left(E_{\mathrm{F}}\right) \frac{1}{2.1} .
$$

It has been checked that $T_{\mathrm{CW}}$ values resulting from Eqs. (11) and (18) agree with those determined by numerical minimization of the free energy, which is obtained by summing up eigenvalues of the corresponding $4 \times 4$ Luttinger matrix. ${ }^{14,42}$ Actually, the theoretical model developed recently, ${ }^{14,42}$ allows one to determine the magnetization as a function of temperature and magnetic field taking into account nonzero anisotropy, finite spin-orbit splitting, and biaxial strain 
within the $6 \times 6$ Luttinger model of the valence band in tetrahedrally coordinated semiconductors. We shall compare results of the $4 \times 4$ and $6 \times 6$ models vis-à-vis our experimental data on $T_{\mathrm{CW}}$, and show that the difference between their predictions is slight for the parameters of $p-\mathrm{Zn}_{1-x} \mathrm{Mn}_{x} \mathrm{Te}$.

\section{Zener versus RKKY model}

We identify two experimentally important situations, for which the Zener model, as introduced above, cases to be valid. The first one corresponds to the case when an average time of carrier tunneling between Mn pairs $\left(V x_{\text {eff }}^{1 / 3}\right)^{-1}$ becomes significantly longer than the inverse exchange energy $\left|\beta N_{0}\right|^{-1}$. Here $V$ is the width of the carrier band, and its magnitude, not the Fermi energy as sometimes suggested, constitutes the relevant energy scale. For long tunneling times, the molecular-field and virtual-crystal approximations break down, an effect detected in $\mathrm{Cd}_{1-x} \mathrm{Mn}_{x} \mathrm{~S} .{ }^{43}$ Doubleexchange model constitutes the appropriate description of the carrier-mediated exchange interaction in the limit $V \rightarrow 0$.

The second case is that of a large carrier concentration, $n>x_{\text {eff }} N_{0}$. In this region, important changes in the carrier response function occur at the length scale of a mean distance between the localized spins. Accordingly, the description of spin magnetization by the continuous-medium approximation, which constitutes the basis of the Zener model, ceases to be valid. In contrast, the RKKY model is a good starting point in this regime, as it provides the dependence of the interaction energy of particular spin pairs as a function of their distance. This makes it possible to evaluate the system energy for a given distribution of the localized spins.

We note in passing that in the limit when the continuousmedium approximation is valid, $n \gg x_{\text {eff }} N_{0}$, the mean-field value of the ordering temperature $T(\mathbf{q})$ deduced from the Zener and RKKY model are identical, independently of microscopic spin arrangement. In particular, in both models, the Curie temperature is determined entirely by matrix elements that are diagonal in $\mathbf{k}$, as nondiagonal terms vanish when the continuous-medium approximation is valid. If this is not the case, the two models are equivalent only if the spins are randomly distributed over a continuum. ${ }^{3}$

Since the RKKY model appears as more general, it is tempting to adopt it for the description of experimental findings. Unfortunately, however, in the presence of spin-orbit interaction, the spin-spin Hamiltonian contains nonscalar pseudo-dipole and Moriya-Dzialoshinskii terms, whose dependence of the pair distance seems to be described by nonelementary functions. Briefly, the RKKY model is technically much more cumbersome than the Zener model for the holes in the $\Gamma_{8}$ band. On the other hand, in the case of the sample with the highest doping level, the hole concentration $p=1.2 \times 10^{20} \mathrm{~cm}^{-3}$ becomes comparable with $x_{\mathrm{eff}} N_{0}$ $=1.8 \times 10^{20} \mathrm{~cm}^{-3}$. Moreover, we note that the blocking of n.n. Mn pairs in the zero-spin state by antiferromagnetic superexchange not only reduces the effective spin concentration but also makes the spin distribution to be highly nonrandom-no n.n. Mn pairs are involved in the ferromagnetic interactions.
In order to evaluate the resulting effect on the CurieWeiss temperature we start from the expression for the energy of the RKKY exchange interaction between two Mn spins at relative position $\mathbf{R}$ induced by carriers described by $S$-like wave functions (see, e.g., Ref. 3),

$$
J(\mathbf{R})=\chi_{h}(0) \frac{2 k_{\mathrm{F}}^{3}}{\pi} \beta^{2} \frac{\sin \left(2 k_{\mathrm{F}} R\right)-2 k_{\mathrm{F}} R \cos \left(2 k_{\mathrm{F}} R\right)}{\left(2 k_{\mathrm{F}} R\right)^{4}} .
$$

Since the main contribution to $\widetilde{\chi}_{h}(\mathbf{q})$ in Eq. (15) comes from the heavy hole band, we use the heavy hole wave vector at the Fermi level for $k_{\mathrm{F}}$ in Eq. (19). At the same time, the prefactor in Eq. (19), the hole spin susceptibility $\tilde{\chi}_{h}$ at $q$ $=0$, is calculated from Eq. (16), so that it takes the complex structure of the $\Gamma_{8}$ bands into account.

The mean-field value of ferromagnetic temperature $T_{F}$ is then obtained from the first moment of the distribution of the pair interaction energies $J\left(R_{i j}\right)$. Thus, we determine $T_{\mathrm{F}}$ by a summation of the interaction energies $J(R)$ between a given spin at $R=0$, and all other free Mn spins distributed over the cation sites of the zinc-blende lattice,

$$
T_{\mathrm{F}}=\frac{S(S+1)}{3 k_{B}} \sum_{\text {fcc sites }} P(i) J\left(\mathbf{R}_{i}\right) .
$$

Here, the occupation probability is $P(i)=0$ for the nearest neighbors to the origin (since the point $R=0$ is occupied by the Mn spin, the n.n. spin would be blocked antiferromagnetically), and $P(i)=x_{\text {eff }}$ for all other fcc sites. As mentioned above, replacing the discrete sum in Eq. (20) by a continuous integration with the uniform density $x_{\text {eff }}$ would lead to Eq. (11). We shall compare those two models for $T_{\mathrm{F}}$ as well as their ability to describe the experimental data in Sec. V. Finally, we note that on increasing the carrier concentration the second moment of the distribution $J\left(R_{i j}\right)$ grows faster than the first moment. The former determines the spin-freezing temperature $T_{g}$, which at some point may become higher than $T_{\mathrm{F}}$. We evaluate that $T_{g}<T_{\mathrm{F}}$ for the samples studied here. Such a cross-over from the ferromagnetic to spin-glass phase as a function of $p / x N_{0}$ has, in fact, been observed in IV-VI DMS. ${ }^{44}$ For even greater values of $p / x N_{0}$, the Kondo temperature $T_{K}$ may become higher than $T_{g}$, so that screening of the spins by the carriers will occur at $T<T_{K}$ if $T_{K}>T_{g}$.

\section{Effects of disorder and carrier-carrier interactions}

The present experimental results address the question of how disorder in the electronic subsystem affects the carriermediated interaction among the localized spins. In the framework of the RKKY model, the mean free path $l$ sets an upper distance of Mn pairs contributing to the first moment of the distribution $J\left(R_{i j}\right)$. This leads to a reduction of $T_{\mathrm{F}}$ by (1 $\left.-\pi / 4 k_{\mathrm{F}} l\right)$ for $k_{\mathrm{F}} l \gg 1$. $^{3}$ In terms of the Zener model, this reduction factor represents the scattering broadening of the thermodynamic density-of-states $\rho\left(E_{\mathrm{F}}\right){ }^{3,45}$ At the same time, the second moment, and thus $T_{g}$, is only affected by spin-dependent scattering. ${ }^{46}$ 
Interestingly enough, neither $l$ nor $\rho\left(E_{\mathrm{F}}\right)$ exhibit critical behavior at the MIT-typically $l \approx k_{\mathrm{F}}^{-1}$ at criticality when approaching the MIT from the metallic side. In contrast, the localization length $\xi$ diverges at the MIT and, according to the scaling picture, decreases gradually towards the Bohr radius $a^{*}$ deeply in the insulator phase. Then, the ferromagnetic exchange is mediated by carriers thermally excited to the band ${ }^{16}$ or by spin-dependent coupling between BMP. ${ }^{17}$ However, over a wide range of dopant concentrations, the average value of $\xi$ is significantly larger than both $a^{*}$ and $l$. We expect that such weakly localized carriers carry efficiently the spin-spin coupling. Since distances smaller than $l$ are important for ferromagnetism, carrier localization will have a minor influence on the value of $T_{\mathrm{F}}$ for such an exchange mechanism.

At the same time, however, large mesoscopic fluctuations in the local values of $\rho\left(E_{\mathrm{F}}\right)$ and $\xi$, are expected in the vicinity of the MIT. This will introduce additional randomness in the system, the corresponding correlation length being of the order of either spin coherence, thermal or localization length, each presumably much longer than that characterizing magnetic disorder. In a simplistic two-fluid picture, ${ }^{14,42}$ we envisage that mosaics of ferromagnetic and paramagnetic regions appear at the Curie temperature. The latter corresponds to regions not visited by the carriers. The former contain delocalized or weakly localized carriers. Such carriers set a longrange ferromagnetic correlation between the Mn spins, including those contributing to BMP that are formed around singly occupied impurity-like states. According to this model, the ferromagnetic portion of the material, and thus the magnitude of spontaneous magnetization, grows with the dopant concentration.

Finally, we consider the enhancement of the tendency towards ferromagnetism by the carrier-carrier interactions. Within the Zener model, this enhancement is described by the Fermi liquid parameter $A_{\mathrm{F}}$. The value $A_{\mathrm{F}} \approx 1.2$ was calculated by a local spin-density approach for a $3 \mathrm{D}$ carrier liquid of similar density. ${ }^{13}$ We adopt this value here, as its enhancement by disorder is probably unimportant due to efficient spin-flip scattering in our system.

\section{COMPARISON OF EXPERIMENTAL AND THEORETICAL RESULTS}

In order to compare experimental and theoretical results for samples with various Mn content $x$ and hole concentrations $p$, we introduce a normalized value of the ferromagnetic temperature,

$$
\widetilde{T}_{\mathrm{F}}=\left(T_{\mathrm{CW}}+T_{\mathrm{AF}}\right) / 10^{2} x_{\mathrm{eff}},
$$

where $T_{\mathrm{CW}}$ and $x_{\text {eff }}$ are determined from the measurements of the magnetic susceptibility as a function of temperature, whereas the corresponding values of $T_{\mathrm{AF}}$ are calculated from Eq. (5). The actual values of $T_{\mathrm{CW}}$ and $x_{\text {eff }}$ for the studied samples are summarized in Table I (Sec. II). According to

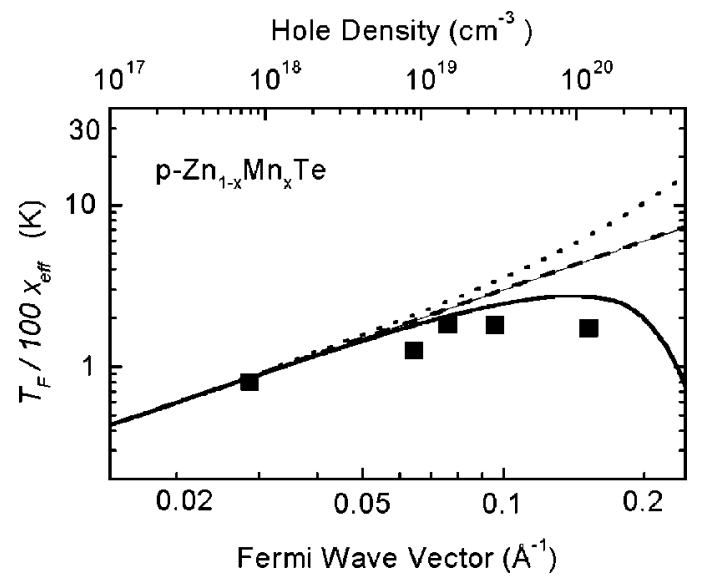

FIG. 9. Experimental (symbols) and calculated (lines) normalized ferromagnetic temperature, $T_{\mathrm{F}} / 10^{2} x_{\text {eff }}$, vs. the wave vector at the Fermi level. The corresponding hole densities are indicated in the top scale. Dashed line: Zener model with the hole dispersion calculated from the $4 \times 4$ Luttinger spherical model for the $\Gamma_{8}$ band; dotted line: Zener model including the coupling between the $\Gamma_{8}$ and $\Gamma_{7}$ bands $(6 \times 6$ Luttinger model, Ref. 14); solid line: the RKKY and $6 \times 6$ Luttinger model for $x_{\text {eff }}=0.015$, taking into account the effect of the antiferromagnetic interactions on statistical distribution of unpaired Mn spins.

the Zener model [Eq. (11)], the normalized ferromagnetic temperature, as defined by Eq. (21), does not depend on the Mn content.

Figure 9 presents experimental and theoretical values of $\widetilde{T}_{\mathrm{F}}$ as a function of $p$. The studied hole concentration range covers both sides of the MIT, the sample with the highest $p$ values being metallic. Remarkably, the presence of the MIT appears to have no effect on the experimental values of $\widetilde{T}_{\mathrm{F}}(p)$. This substantiates the view that the two phenomena, carrier-mediated ferromagnetic interactions and carrier localization, are sensitive to carrier wave functions at different length scales: the former shorter while the latter longer than the mean free path. This encourages us to interpret the data disregarding localization effects. Furthermore, we shall neglect the influence of scattering on the density-of-states, an approximation that should be relaxed once appropriate information would be available.

The dotted line in Fig. 9 represents results of the analytic calculation within the Zener model, employing $4 \times 4$ spherical Luttinger Hamiltonian, Eqs. (11) and (16). Similarly, the dashed line was obtained numerically from the $6 \times 6$ model. ${ }^{14,42}$ Due to the large magnitude of spin-orbit splitting in $\mathrm{ZnTe}$, the difference between the models is slight, even for relatively large hole concentrations. It is seen that the Zener model describes correctly the ferromagnetic temperature in the region of low hole concentrations, but it predicts a significantly too large value of $\widetilde{T}_{\mathrm{F}}$ for the sample with $p$ $=1.2 \times 10^{20} \mathrm{~cm}^{-3}$. As already mentioned, in this sample $x_{\text {eff }}=0.015$, so that the inverse Fermi wave vector becomes comparable to the mean Mn-Mn distance, $d_{\mathrm{Mn}-\mathrm{Mn}} k_{\mathrm{F}}=1.4$. As a result the continuous-medium approximation, inherent to the Zener model, ceases to be valid. The solid line in Fig. 9 depicts numerical data obtained within the RKKY model, 

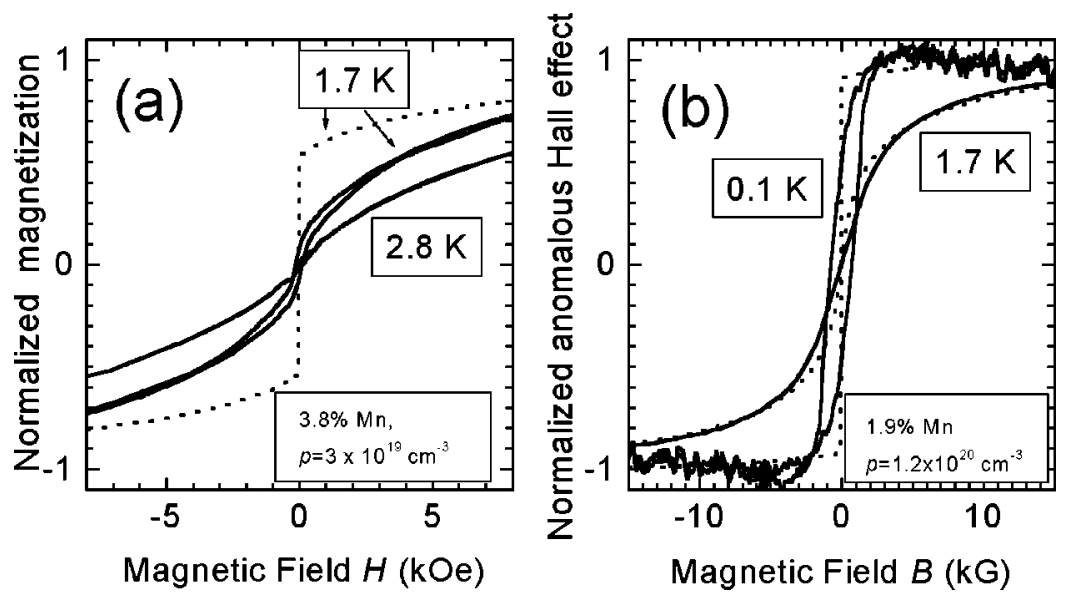

FIG. 10. Low-temperature magnetization as a function of the magnetic field measured directly for nonmetallic $p-\mathrm{Zn}_{0.962} \mathrm{Mn}_{0.038} \mathrm{Te}$ sample (a) and determined from the Hall data for metallic $p-\mathrm{Zn}_{0.981} \mathrm{Mn}_{0.019} \mathrm{Te}$ sample (b), whose magnetic susceptibilities are shown in Figs. 6 and 4, respectively. The dotted lines present results of the mean-field calculation, which indicate that only a part of Mn spins contribute to ferromagnetic order in the insulating sample.
Eqs. (19) and (20) for $x_{\text {eff }}=0.015$, with the long-wavelength hole spin susceptibility computed by the $6 \times 6$ Luttinger model. A significant reduction of $\widetilde{T}_{\mathrm{F}}$ for large $p$, consistent with the experimental findings, is clearly visible. This reduction reflects the important effect of the antiferromagnetic n.n. Mn pairs on the distribution of the free spins. In view that theory is developed with no adjustable parameters, we conclude that the main processes accounting for the magnitude of the Curie-Weiss temperature are well understood in $p-\mathrm{Zn}_{1-x} \mathrm{Mn}_{x} \mathrm{Te}$.

Figure 10 presents the dependence of magnetization on the magnetic field for one nonmetallic and one metallic sample. The dependence of magnetization on the magnetic field is in accord with the expectation of the mean-field theory in the case of the metallic sample. In contrast, the magnitude of magnetization observed in the non-metallic sample in the weak fields is about two times smaller than expected. This substantiates the conjecture about the phase separation into the regions with a different degree of Mn spin polarization on the insulator side of the MIT.

\section{SUMMARY AND OUTLOOK}

The progress in nitrogen doping of $\mathrm{Zn}_{1-x} \mathrm{Mn}_{x}$ Te by MBE achieved in course of this work has made it possible to grow II-VI DMS, in which hole kinetic energy is high enough to over-compensate electrostatic and magnetic disorder, so that the metal phase exists down to the millikelvin temperature range. In such a sample the doping-induced ferromagnetic ordering has been put into the evidence. Two factors have been identified, which make the Curie temperature relatively low, $T_{C} \approx 1.5 \mathrm{~K}$ at $p=1.2 \times 10^{20} \mathrm{~cm}^{-3}$ and $x=0.019$, despite the rather large values of the effective mass and $p$ - $d$ exchange integral. First, the spin-orbit interaction in the valence band leads to more than two-fold reduction of $T_{C}$. Second, the superexchange antiferromagnetic interactions have been found to lower $T_{C}$ even further. In particular, the residual antiferromagnetic interactions between more distant Mn pairs contribute to the reduction of $T_{C}$. Moreover, the presence of magnetically inert nearest-neighbor Mn pairs not only lowers the effective Mn concentration, but also makes the antiferromagnetic portion of the RKKY interaction to become more significant. The resulting competition between the ferromagnetic and antiferromagnetic interactions is expected to grow with $p / x$, and may ultimately result in the transition to a spin-glass phase. Alternatively, with decreasing $x$ at given $p$, the Kondo effect may show up.

We note that the present results, as well as those for $\mathrm{Ga}_{1-x} \mathrm{Mn}_{x} \mathrm{As},{ }^{14,42}$ make it possible to single out dominant effects accounting for differences between temperatures of ferromagnetic ordering in particular families of magnetic semiconductors. ${ }^{14,42}$ One of the effects is the magnitude of the spin-orbit splitting $\Delta_{o}$ in the valence band, which-if much greater than the Fermi energy-reduces $T_{C}$. This is the case of $p-\mathrm{Zn}_{1-x} \mathrm{Mn}_{x} \mathrm{Te}$. In contrast, due to the smaller value of $\Delta_{o}$ in $\mathrm{Ga}_{1-x} \mathrm{Mn}_{x} \mathrm{As}$, the reduction ceases to be important. Moreover, the mixing between $\Gamma_{8}$ and $\Gamma_{7}$ bands enlarges the density-of-states at the Fermi level. Another important aspect of III-V DMS is associated with the fact that Mn ions supply both spins and holes. This results in a large Coulomb potential at closely lying Mn pairs. Accordingly, such a complex binds a hole, which mediates a strong ferromagnetic coupling that overcompensates the intrinsic antiferromagnetic interaction. As a result, the strong reduction of $T_{C}$ by the superexchange observed in II-VI semiconductors is virtually absent in the case of III-V DMS. ${ }^{14}$

Particularly interesting is the problem of interplay between Anderson-Mott localization and carrier-mediated exchange interaction. Our results demonstrate the presence of magnetoresistance and temperature dependent localization, qualitatively similar to those observed previously in $n$-type DMS, but somewhat enhanced due to a greater magnitude of the exchange energy in the case of the holes. At the same time, the strength of the carrier-mediated ferromagnetic interaction appears to be insensitive to hole localization. This behavior, observed also in $\mathrm{Ga}_{1-x} \mathrm{Mn}_{x} \mathrm{As},{ }^{2}$ is assigned to the different length scales involved in the two processes. However, the low-temperature phase in nonmetallic samples show a number of peculiarities, such as slow dynamics and partial saturation of the magnetization. Furthermore, in contrast to, e.g., Eu chalcogenides, no re-entrance to the metallic behavior is observed in the low-temperature phase, which indicates that spin-disorder scattering remains effective. Thus, the ground state on the insulator side of the MIT may not be a simple colinear ferromagnet. The presence of a 
phase separation into ferromagnetic and paramagnetic spin puddles has been suggested ${ }^{14}$ to occur near the MIT. We plan to employ local experimental probes in order to verify this conjecture. We also hope that our results will prompt a theoretical examination of effects of mesoscopic fluctuations in the density-of-states and localization radius upon the carrier-mediated ferromagnetic interactions between magnetic impurities.

\section{ACKNOWLEDGMENTS}

We thank Jacek Furdyna, Hideo Ohno, and Maciej Sawicki for valuable discussions. The Grenoble-Warsaw collaboration was supported by the "Polonium" Program, the work in Poland by the Foundation for Polish Science and the State Committee for Scientific Research under Grant No. 2-PB03B-02417.
*Electronic address: cibert@ drfmc.ceng.cea.fr

†Present address: IEF, UMR 6822 CNRS, Université Paris-Sud, F-91405 Orsay Cédex, France.

†Electronic address: dietl@ifpan.edu.pl; URL: www.ifpan.edu.p1/ SL-2/s123.html.

${ }^{1}$ T. Story, R. R. Galązka, R. B. Frankel, and P. A. Wolff, Phys. Rev. Lett. 56, 777 (1986); P. Łazarczyk, T. Story, M. Arciszewska, and R. R. Gałązka, J. Magn. Magn. Mater. 169, 151 (1997), and references cited therein.

${ }^{2}$ F. Matskura, H. Ohno, A. Shen, and Y. Sugawara, Phys. Rev. B 57, R2037 (1998); H. Ohno, Science 281, 951 (1998), and references cited therein.

${ }^{3}$ T. Dietl, A. Haury, and Y. Merle d'Aubigné, Phys. Rev. B 55, R3347 (1997); T. Dietl, J. Cibert, D. Ferrand, and Y. Merle d'Aubigné, Mater. Sci. Eng., B 63, 103 (1999).

${ }^{4}$ A. Haury, A. Wasiela, A. Arnoult, J. Cibert, S. Tatarenko, T. Dietl, and Y. Merle d'Aubigné, Phys. Rev. Lett. 79, 511 (1997).

${ }^{5}$ D. Ferrand, J. Cibert, C. Bourgognon, S. Tatarenko, A. Wasiela, G. Fishman, A. Bonanni, H. Sitter, S. Koleśnik, J. Jaroszyński, and T. Dietl, 9th International Conference on II-VI Compounds, Kyoto, 1999, J. Cryst. Growth 214/215, 387 (2000).

${ }^{6}$ M. Oestreich, J. Hubner, D. Hagele, P. J. Klar, W. Heimbrodt, W. W. Ruhle, D. E. Ashenford, and B. Lunn, Appl. Phys. Lett. 74, 1251 (1999).

${ }^{7}$ R. Fiederling, M. Keim, G. Reuscher, W. Ossau, G. Schmidt, A. Waag, and L. W. Molenkamp, Nature (London) 402, 787 (1999).

${ }^{8}$ Y. Ohno, D. K. Young, B. Beschoten, F. Matskura, H. Ohno, and D. D. Awschalom, Nature (London) 402, 790 (1999).

${ }^{9}$ J. K. Furdyna, J. Appl. Phys. 64, R29 (1988); T. Dietl, in: Handbook on Semiconductors, edited by T. S. Moss (North-Holland, Amsterdam, 1994), Vol. 3b, p. 1251.

${ }^{10}$ D. Loss and D. Di Vincenzo, Phys. Rev. A 57, 120 (1998); D. Di Vincenzo, Science 270, 255 (1995).

${ }^{11}$ H. Ohno, H. Munekata, T. Penney, S. von Molnàr, and L. L. Chang, Phys. Rev. Lett. 68, 2664 (1992).

${ }^{12}$ H. Ohno, A. Shen, F. Matsukura, Y. Sugawara, A. Endo, A. Oiwa, S. Katsumoto, and Y. Iye, Appl. Phys. Lett. 69, 363 (1996); H. Ohno, J. Magn. Magn. Mater. 200, 110 (1999), and references cited therein.

${ }^{13}$ T. Jungwirth, W. A. Atkinson, B. H. Lee, and A. H. MacDonald, Phys. Rev. B 59, 9818 (1999).

${ }^{14}$ T. Dietl, H. Ohno, J. Cibert, F. Matskura, and D. Ferrand, Science 287, 1019 (2000).

${ }^{15}$ See, for example, J. A. Mydosh, Spin Glasses (Taylor \& Francis, London, 1993).

${ }^{16}$ E. A. Pashitskii and S. M. Ryabchenko, Fiz. Tverd. Tela (S.Peterburg) 21, 545 (1979) [Sov. Phys. Solid State 21, 322 (1979)].
${ }^{17}$ P. A. Wolff, R. N. Bhatt, and A. C. Durst, J. Appl. Phys. 79, 5196 (1996); R. N. Bhatt and Xin Wan, Int. J. Mod. Phys. C 10, 1459 (1999).

${ }^{18}$ T. R. Kirkpatrick and D. Belitz, e-print: xxx.lanl.gov/cond-mat/9912477; Phys. Rev. B in press; e-print: xxx.lanl.gov/cond-mat/9912478; Phys. Rev. B, in press.

${ }^{19}$ B. N. Narozhny, I. L. Aleiner, and A. I. Larkin, e-print: xxx.lanl.gov/cond-mat/0005267.

${ }^{20}$ M. Grün, A. Haury, J. Cibert, and A. Wasiela, J. Appl. Phys. 79, 7386 (1996); T. Baron, K. Saminadayar, and N. Magnea, ibid. 83, 1354 (1998).

${ }^{21}$ A. Baldereschi and N. O. Lipari, Phys. Rev. B 8, 2697 (1973); N. O. Lipari and M. Altarelli, ibid. 32, 5138 (1985).

${ }^{22}$ H. P. Wagner, S. Lankes, K. Wolf, D. Lichtenberger, W. Kuhn, P. Link, and W. Gebhardt, J. Lumin. 52, 41 (1992).

${ }^{23}$ D. Ferrand, C. Bourgognon, J. Cibert, A. Wasiela, S. Tatarenko, Y. Merle d'Aubigné, A. Bonanni, D. Stifter, H. Sitter, Le Van Khoi, S. Koleśnik, J. Jaroszyński, M. Sawicki, T. Andrearczyk, and T. Dietl, 22nd International Conference on Low Temperature Physics, Helsinki 1999, Physica B 284-288, 1177 (2000).

${ }^{24}$ D. Ferrand, J. Cibert, A. Wasiela, C. Bourgognon, S. Tatarenko, G. Fishman, S. Koleśnik, J. Jaroszyński, T. Dietl, B. Barbara, and D. Dufeu, 44th Magnetism and Magnetic Materials Conference, San Jose 1999, J. Appl. Phys. 87, 5461 (2000).

${ }^{25}$ M. Sawicki, T. Dietl, J. Kossut, J. Igalson, T. Wojtowicz, and W. Plesiewicz, Phys. Rev. Lett. 56, 508 (1986).

${ }^{26}$ Y. Shapira, N. F. Oliveira Jr., D. H. Ridgley, R. Kershaw, K. Dwight, and A. Wold, Phys. Rev. B 34, 4187 (1986); Y. Shapira, N. F. Oliveira Jr., P. Becla, and T. K. Wu, 41, 5931 (1990).

${ }^{27}$ P. Głód, T. Dietl, M. Sawicki, and I. Miotkowski, Physica B 194-196, 995 (1994).

${ }^{28}$ T. Wojtowicz, T. Dietl, M. Sawicki, W. Plesiewicz, and J. Jaroszyński, Phys. Rev. Lett. 56, 2419 (1986).

${ }^{29}$ P. Leroux-Hugon and A. Ghazali, J. Phys. C 5, 1072 (1972); J. N. Chazalviel, Phys. Rev. B 11, 3918 (1975).

${ }^{30}$ J. A. Gaj, R. Planel, and G. Fishman, Solid State Commun. 29, 435 (1979).

${ }^{31}$ G. Barilero, C. Rigaux, M. Menant, Nguyen Hy Hau, and W. Giriat, Phys. Rev. B 32, 5144 (1985); Y. Shapira, N. F. Oliveira, D. H. Ridgley, R. Kershaw, K. Dwight, and A. Wold, 34, 4187 (1986); J. P. Lascaray, A. Bruno, M. Nawrocki, J. M. Broto, J. C. Ousset, S. Askenazi, and R. Triboulet, 35, 6860 (1987); A. Twardowski, C. J. M. Denissen, W. J. M. de Jonge, A. T. A. M. de Waele, M. Demianiuk, and R. Triboulet, Solid State Commun. 59, 199 (1986).

${ }^{32}$ J. A. Gaj, W. Grieshaber, C. Bodin, J. Cibert, G. Feuillet, Y. Merle d'Aubigné, and A. Wasiela, Phys. Rev. B 50, 5512 (1994). 
${ }^{33}$ Y. Shapira, S. Foner, D. H. Ridgley, K. Dwight, and A. Wold, Phys. Rev. B 30, 4021 (1984).

${ }^{34}$ J. M. Fatah, T. Piorek, P. Harrison, T. Stirner, and W. E. Hagston, Phys. Rev. B 49, 10341 (1994).

${ }^{35}$ M. E. Fisher, S.-k. Ma, and B. G. Nickel, Phys. Rev. Lett. 29, 917 (1972).

${ }^{36}$ J. A. Gaj, J. Ginter, and R. R. Gałązka, Phys. Status Solidi B 89, 655 (1978).

${ }^{37}$ A. Twardowski, P. Swiderski, M. von Ortenberg, and R. Pauthenet, Solid State Commun. 50, 509 (1984).

${ }^{38}$ C. Zener, Phys. Rev. 81, 440 (1950); ibid. 83, 299 (1950); H. Frölich and F. R. N. Nabarro, Proc. R. Soc. London, Ser. A 175, 382 (1940).

${ }^{39}$ J. Cibert, P. Kossacki, A. Haury, D. Ferrand, A. Wasiela, Y. Merle d'Aubigné, A. Arnoult, S. Tatarenko, and T. Dietl, in Proceedings of the 24th International Conference on the Physics of Semiconductors, Jerusalem, Israel (1998), edited by D. Gershoni (World Scientific, Singapore, 1999), p. 51.

${ }^{40}$ T. Dietl, J. Cibert, D. Ferrand, and Y. Merle d'Aubigné, Mater. Sci. Eng., B 63, 103 (1999); T. Dietl, J. Cibert, P. Kossacki, D. Ferrand, S. Tatarenko, A. Wasiela, Y. Merle d'Aubigné, F. Matsukura, N. Akiba, and H. Ohno, Physica E (Amsterdam) 7, 967 (2000); T. Dietl and H. Ohno, e-print: xxx.lanl.gov/cond-mat/
0002450.

${ }^{41}$ C. Hermann and C. Weisbuch, in Optical Orientation, Chap. 11, edited by F. Meier and B. P. Zakharchenya (Elsevier, Amsterdam, 1984).

${ }^{42}$ T. Dietl, H. Ohno, and F. Matsukura, e-print: xxx.lanl.gov/cond-mat/0007190.

${ }^{43}$ C. Benoit à la Guillaume, D. Scalbert, and T. Dietl, Phys. Rev. B 46, 9853 (1992).

${ }^{44}$ P. J. T. Eggenkamp, C. W. H. M. Vennix, T. Story, H. J. M. Svagten, C. H. W. Swüste, and W. J. M. de Jonge, J. Appl. Phys. 75, 5728 (1994); P. T. J. Eggenkamp, H. J. M. Swagten, T. Story, V. I. Litvinov, C. H. W. Swüste, and W. J. M. de Jonge, Phys. Rev. B 51, 15250 (1995).

${ }^{45}$ P. Kossacki, D. Ferrand, A. Arnoult, J. Cibert, S. Tatarenko, A. Wasiela, Y. Merle d'Aubigné, J-L. Staehli, J-D. Ganiere, W. Bardyszewski, K. Świątek, M. Sawicki, J. Wróbel, and T. Dietl, Physica E (Amsterdam) 6, 709 (2000).

${ }^{46}$ A. Yu. Zyuzin and B. Z. Spivak, Zh. Eksp. Teor. Fiz. 43, 185 (1986) [JETP Lett. 43, 234 (1986)]; L. N. Bulaevskii and S. V. Panyukov, Pis'ma Zh. Eksp. Teor. Fiz. 43, 190 (1986) [JETP Lett. 43, 240 (1986)]; A. Jagannathan, E. Abrahams, and M. J. Stephen, Phys. Rev. B 37, 436 (1988). 